\title{
Variability among 2D and 3D methods of calculating mitral valve area: a comparative study with pressure half time method
}

\author{
Amit Kumar Agarwal, ${ }^{1}$ Deewakar Sharma, ${ }^{2}$ Sajan Gopal Baidya, ${ }^{2}$ Dipanker Prajapati ${ }^{2}$ \\ National Academy of Medical Sciences, Kathmandu \\ Shahid Gangalal National Heart Centre, Bansbari, Kathmandu
}

Corresponding author: Amit Kumar Agarwal,

Department of Cardiology,

National Academy of Medical Sciences, Bir Hospital, Mahaboudha, Kathmandu

Email address: amit_bjg@hotmail.com

\section{Abstract}

\begin{abstract}
Background and Aims: The aim of this study was to evaluate the feasibility, reproducibility and accuracy of Live Three dimensional Echocardiography (3DE), Two dimensional Echocardiography (2DE) and Three dimensional Xplane Echocardiography (3D Xplane) for the estimation of mitral valve area (MVA) and to assess which method has the best agreement with the MVA non- invasively evaluated by the Pressure half time (PHT) method in isolated rheumatic mitral valve stenosis (RMVS).

Methods: In 40 patients with isolated RVMS in sinus rhythm (29 female) MVA was determined by Doppler PHT method and compared with measurements obtained by 2DE, Live 3DE and 3D Xplane method. All measurements were performed by two independent observers.

Results: For both observers mean MVA was calculated minimum with $3 \mathrm{DE}$ (observer 1: $0.68 \pm 0.19$, observer 2: $0.68 \pm 0.19$ ). Intraobserver variability was least with 3D Xplane method (observer $1 \mathrm{cv} 0.23$, observer $2 \mathrm{cv} 0.23$ ). Although there was no significant interobserver variability for each method, it was least for MVA by 3D Xplane method (difference -0.036) and maximum for 3DE method (-0.098). Difference of each method with PHT showed lowest difference with 3D Xplane (-0.30) and highest with 3DE (-0.63).

Conclusions: TTE 3D Xplane provides accurate and highly reproducible measurements of MVA and can easily be performed from optimal PLAX view and corresponding parasternal short-axis views acquired in the same bisected image using 3D Xplane technique. It was much easier and faster to define the image plane in short axis with the smallest orifice area when 3D Xplane method was used.
\end{abstract}

Key words: 3D Xplane Echocardiography, Live 3D Echocardiography, Mitral valve area, Pressure half time, Planimetry, Rheumatic mitral valve stenosis

DOI: http://dx.doi.org/10.3126/njh.v14i2.18497

Rheumatic mitral valve stenosis (RVMS) still remains an important public health concern in underdeveloped countries. To define the best therapeutic strategy, clinical data and accurate measurements of the mitral valve orifice area are necessary. Two dimensional Echocardiography (2DE) is the most commonly used method for assessment of severity of RVMS. ${ }^{1}$ Mitral valve area (MVA) can be measured by Pressure half time (PHT), 2DE planimetry, continuity equation, and proximal isovelocity surface area methods. ${ }^{2,3} \mathrm{PHT}$ method is affected by changes in preload or left ventricular compliance. Transmitral gradient, continuity equation and cardiac catheterization using Gorlin equation depend upon transvalvular flow and may be affected by cardiac output and the presence of mitral regurgitation. ${ }^{4}$ Although planimetry correlates best with anatomic MVA as assessed by explanted valves, it is highly operator dependent and sometimes laborious. In addition, planimetry requires a parasternal short axis view of the mitral valve and is limited to patients with good image quality from a parasternal window. ${ }^{5}$

Live Three dimensional Echocardiography (3DE) provides a unique "en-face" view of the complete mitral valve apparatus and could, therefore, improve the accuracy of MVA planimetry. ${ }^{6,7}$ However, it has not been routinely performed due to the cumbersome nature of current platforms, prolonged data acquisition, and offline processing time. With the advent of a new transthoracic 3D Xmatrix array probe (Philips Epiq 7) that allows real-time $3 \mathrm{D}$ rendering, many of the above limitations could be circumvented.

The purpose of this study was to determine the accuracy, feasibility and reproducibility of 3DE for calculating MVA in patients with isolated RVMS. In addition, the accuracy of MVA measurement from Live 3DE, 2DE and 3D Xplane method was compared with values obtained by Doppler PHT using it as the gold standard. ${ }^{8}$ 


\section{METHODS}

\section{Study Design}

It was a prospective observational single center study done at Shahid Gangalal National Heart Centre between June 2016 to October 2016.Two independent experienced investigators calculated MVA by four methods (Live 3DE, 2DE, 3D Xplane, PHT) and the average of three consecutive measurements performed by both investigators was used. Both the Observers were blinded for test results.

\section{Study Population}

A total of 51 patients with isolated RVMS were enrolled in the study out of which 11 were excluded from the study and only 40 patients were included in final study. 29 $(72.5 \%)$ were females and $13(32.5 \%)$ were below 20 years. All the patients were planned for Percutaneous transmitral commissurotomy (PTMC). Patients with suboptimal images, heavy mitral valvular calcification, image artifacts precluding the accurate measurement of MVA, Left ventricle dysfunction,severe Aortic regurgitation, atrial fibrillation, multivalvular disease, and prosthetic valve were excluded from the study.

\section{Conventional echo}

2DE and continuous wave Doppler were performed with a Philips Epiq 7 machine with Xmatrix probes. Short axis views of the mitral valve were obtained from the left parasternal window. Careful scanning from the left atrium towards the left ventricle and vice versa identified the smallest orifice of the mitral valve. Particular care was taken to optimize gain settings. Planimetry of the mitral valve area was done by manual tracing of the contours using the diastolic frame with the largest opening amplitude of the mitral valve. Continuous-wave Doppler recordings of transmitral blood flow velocities were obtained in the apical four chamber view. MVA was determined by dividing 220 by the PHT.

\section{Live 3DE}

Live 3DE was performed immediately after the 2DE study. Data were recorded using the aforementioned probe. Live 3DE MVA was performed by obtaining a Live 3D zoom data set. From apical four chamber view from the LA side(surgical view). Multiple cardiac cycles of the mitral valve were recorded using the "zoom" mode (Figure 1). Live 3DE planimetry was performed "en-face" at the ideal cross-section of the mitral valve during its greatest diastolic opening. The ideal cross-section was defined as the most perpendicular view on the plane with the smallest mitral valve orifice. $^{9}$

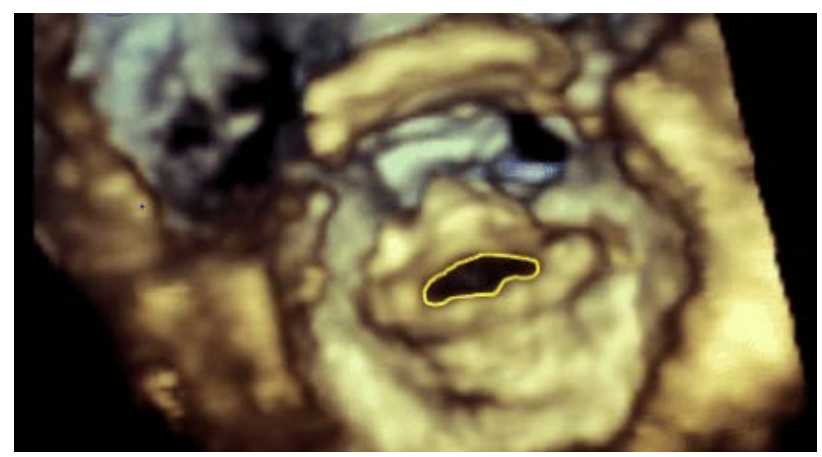

Figure1: live 3DE mitral valve zoomed view from $L A$ side for mitral valve area measurement

\section{D Xplane}

Optimal PLAX and corresponding parasternal short-axis views at mitral valve level were acquired in the same bisected image using 3D Xplane technique.
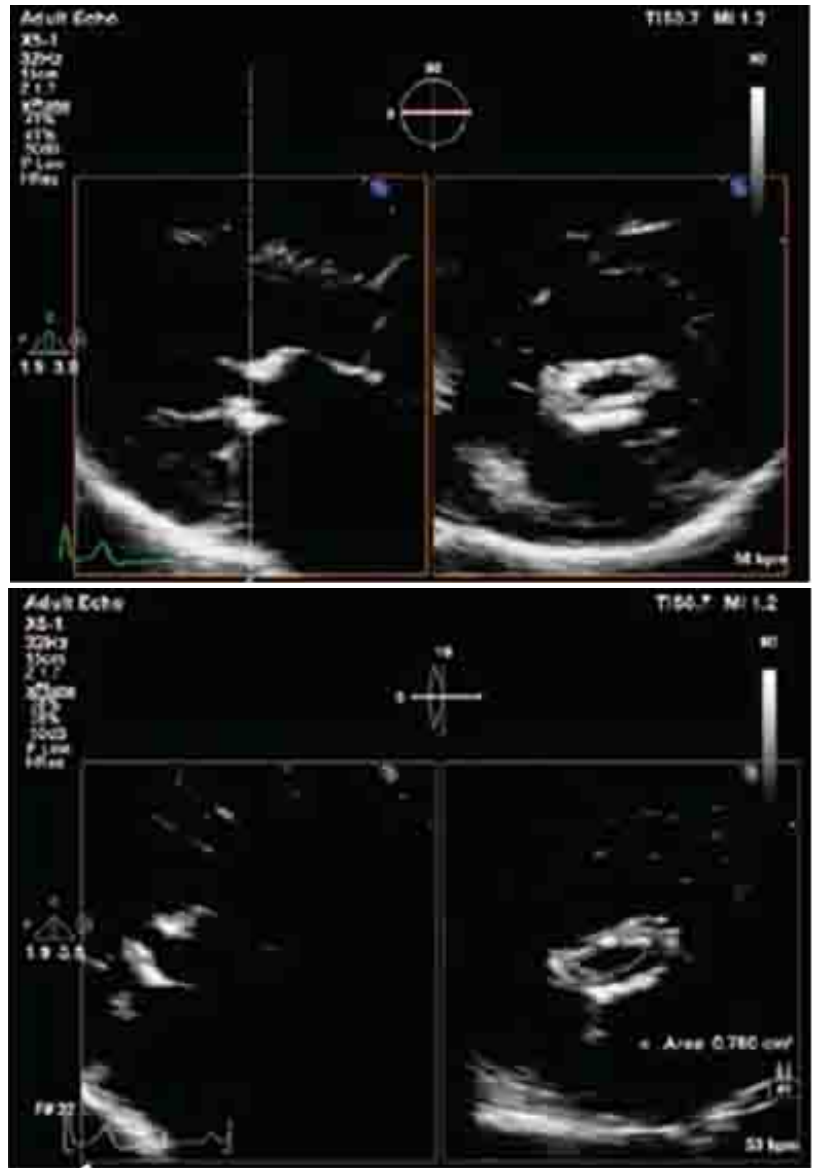

Figure 2: $2 \mathrm{a}$ and $2 \mathrm{~b}$ showing $M V A$ calculation by $3 D$ planimetry with live Xplane assistance.

\section{Statistical Analysis}

Statistical analysis was carried out using SPSS 20(IBM, USA) software. All values are expressed as mean \pm SD. MVA obtained with the different methods was compared by analysis of variance for measurement. Coefficient of variation method was used to look for intraobserver variability for different methods of MVA calculation. Bland-Altman method was used to measure agreement between two metric continuous variables( interobserver variability). The limits of agreement between MVA obtained from the 3DE data set, 3D Xplane method and 2DE planimetric methods with values obtained by Doppler PHT were assessed by difference in different tests.

\section{RESULTS}

Fourty patients with RMVS comprised our study group. Of total patients $13(33 \%)$ were below 20 yrs, 16(40\%) between 20 to 40 yrs and 11(27\%) above 40 yrs. Males were $11(27.5 \%)$ and rest were females $29(72.5 \%)$.

\section{Intraobserver and Interobserver variability of mitral valve area measurements}

For observer 1 mean MVA by 2D planimetry was $1.00 \pm .27$, PHT $0.97 \pm 0.23$, 3D Xplane $0.92 \pm 0.21$ and $3 \mathrm{DE} 0.68 \pm 0.19$ and for second observer mean MVA by $2 \mathrm{D}$ planimetry was $1.00 \pm .26$, PHT $0.95 \pm 0.22,3 \mathrm{D}$ Xplane $0.92 \pm 0.22$ and $3 \mathrm{DE} 0.68 \pm 0.19$. MVA by $3 \mathrm{DE}$ was underestimated as compared to other methods of MVA measurements. We tested for intraobserver variability of MVA for observer 1 and 2 by coefficient of variation and consistency test. For observer 1 MVA by 3D Xplane and PHT method was the less variable( $\mathrm{cv}$ 
$0.23,0.24)$ method and more consistent $(1-\mathrm{cv} 0.75,0.76)$ were as MVA by $2 \mathrm{DE}$ and $3 \mathrm{DE}$ were more variable (cv $0.27,0.28$ ) and less consistent $(1-\mathrm{cv} 0.73,0.71)$. For second observer also MVA by 3D Xplane and PHT method was the less variable( cv
$0.23,0.24)$ method and more consistent $(1-\mathrm{cv} 0.76,0.75)$ were as MVA by $2 \mathrm{DE}$ and $3 \mathrm{DE}$ were more variable (cv $0.25,0.28$ ) and less consistent $(1-\mathrm{cv} 0.74,0.71)$. The same results have been shown by scatter plot diagram in fig .3

Table1: Intraobserver variability for each method was tested by Bland- Altman method

$\begin{array}{llllllll} & \text { N } & \text { Minimum } & \text { Maximum } & \text { Mean } & \text { Std. Deviation } & \text { cv } & 1-c v \\ \text { MVA 2D_1st } & 40 & 0.5000 & 1.5400 & 1.005750 & 0.2710274 & 0.269478 & 0.730522 \\ \text { MVA PHT_1st } & 40 & 0.4000 & 1.3000 & 0.971000 & 0.2362940 & 0.243351 & 0.756649 \\ \text { MVA Xp_1st } & 40 & 0.5000 & 1.2000 & 0.925250 & 0.2189835 & 0.236675 & 0.763325 \\ \text { MVA 3D_1st } & 40 & 0.3000 & 1.0000 & 0.688650 & 0.1959897 & 0.28460 & 0.7154 \\ \text { MVA2D_2nd } & 40 & 0.5000 & 1.4500 & 1.006500 & 0.2607342 & 0.25905 & 0.74095 \\ \text { MVAPHT_2nd } & 40 & 0.3800 & 1.2000 & 0.956750 & 0.2278595 & 0.23816 & 0.76184 \\ \text { MVAXP_2nd } & 40 & 0.4000 & 1.2000 & 0.925500 & 0.2247728 & 0.242866 & 0.757134\end{array}$

Table 2: Interobserver difference in MVA calculation by two observer by Bland Altman method

\begin{tabular}{lllllll} 
& Mean & $\begin{array}{l}\text { Std. } \\
\text { Deviation }\end{array}$ & $\begin{array}{l}\text { Std. Error } \\
\text { Mean }\end{array}$ & \multicolumn{2}{l}{ 95\% Confidence Interval of the Difference } \\
& & & & Lower & Upper & Difference \\
\hline MVA2D_1st-MVA2D_2nd & -0.000750 & 0.1319555 & 0.0208640 & -.0429514 & 0.041451 & -0.0844028 \\
& & & & & & \\
\hline $\begin{array}{l}\text { MVAPHT_1st - } \\
\text { MVAPHT_2nd }\end{array}$ & 0.014250 & 0.0636452 & 0.0100632 & -.0061047 & 0.034604 & -0.0407094 \\
MVAXp1st - MVAXp_2nd & -0.000250 & 0.0573110 & 0.0090617 & -.0185789 & 0.018078 & -0.0366579 \\
\hline MVA3D_1st - MVA3D_2nd & 0.006800 & 0.1541965 & 0.0243806 & -.0425144 & 0.056114 & -0.0986289
\end{tabular}

\section{MVA2d}

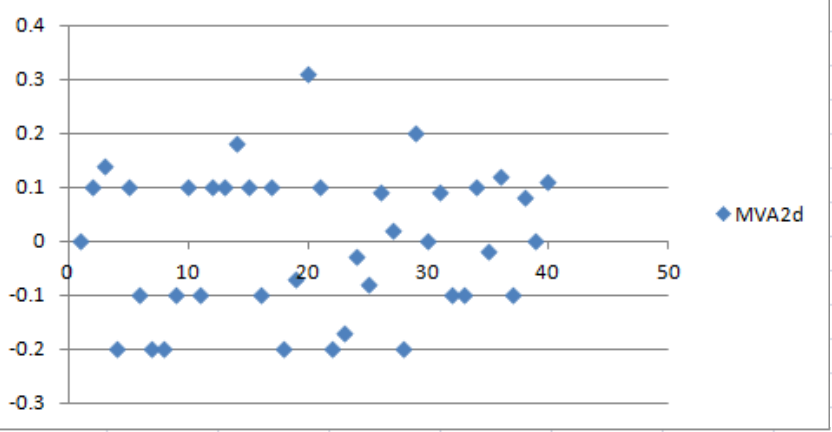

\section{MVA3d}

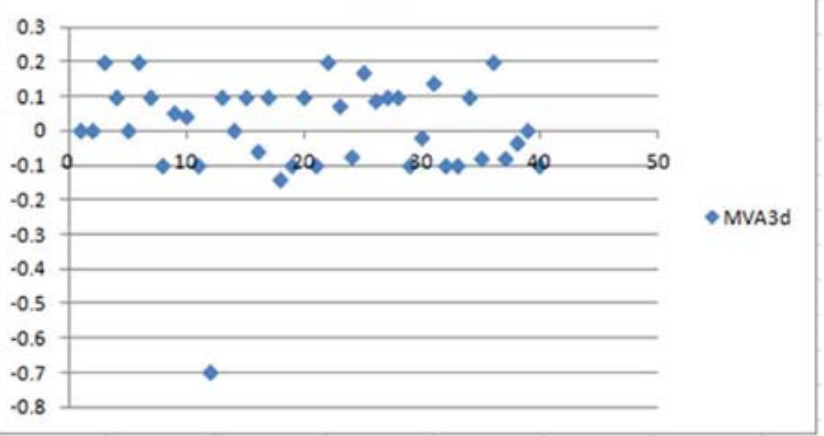

Figure 3: 3a,3b, 3c and 3d scatter plot diagram showing $M V A$ calculation by different method and intra observer variability
MVAPHT

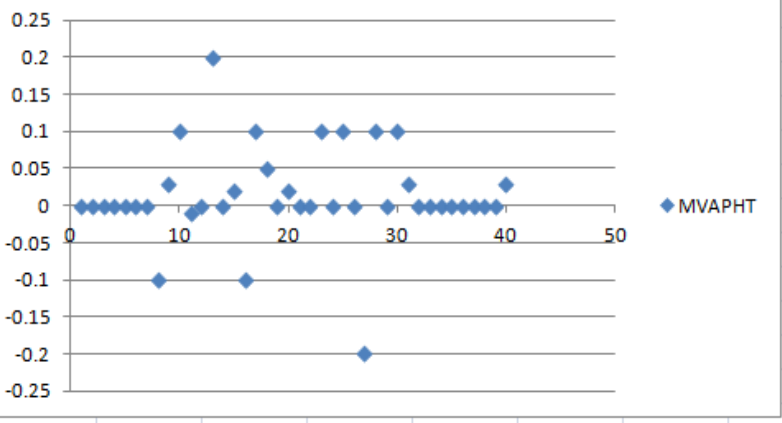

MVAXP

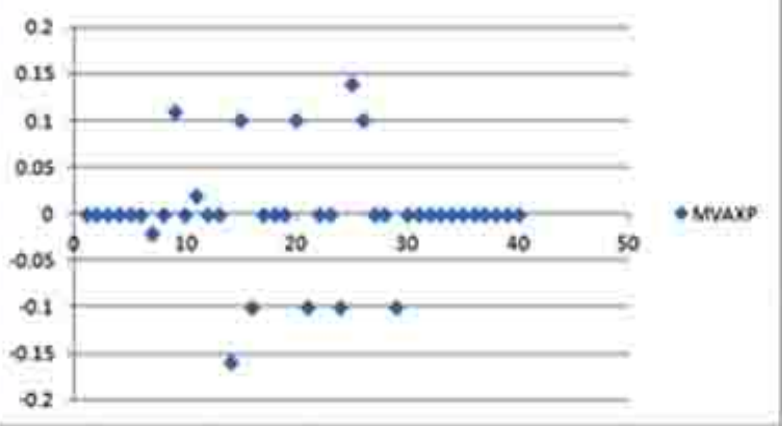


For each method mean difference and SD was computed and $95 \%$ CI of the differences was also calculated, then the lower limit for each method was deducted from upper limit to find the difference. Although there was no significant interobserver variability for each method it was least for MVA by 3D Xplane method( difference -0.036) and maximum for 3DE method $(-0.098)$ (Table2).

\section{DE, 2DE and 3D Xplane Versus Doppler PHT method for MVA measurement}

The difference of each method was deducted to difference of PHT for each observer and then the difference and 2SD was computed. Although there was no significant difference in each method with respect to PHT, the lowest difference was found with 3 DXplane $(-0.30)$ and highest with $3 \mathrm{DE}(-0.63)$. (Table 3 ) The same results have been shown by scatter plot diagram in fig. 4

\section{D-PHT}

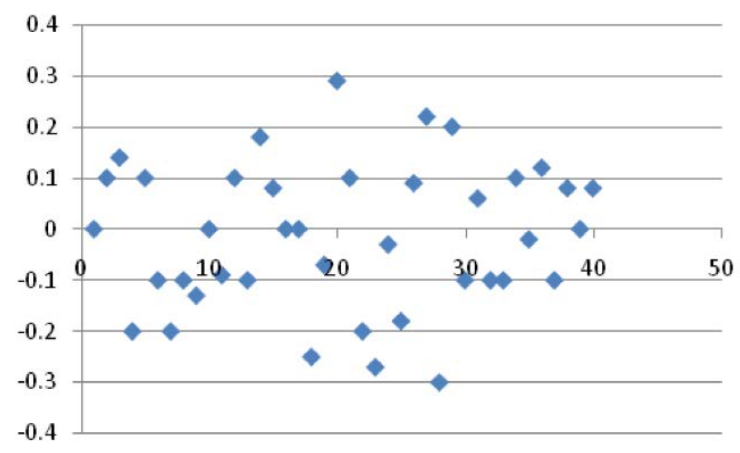

xp-PHT

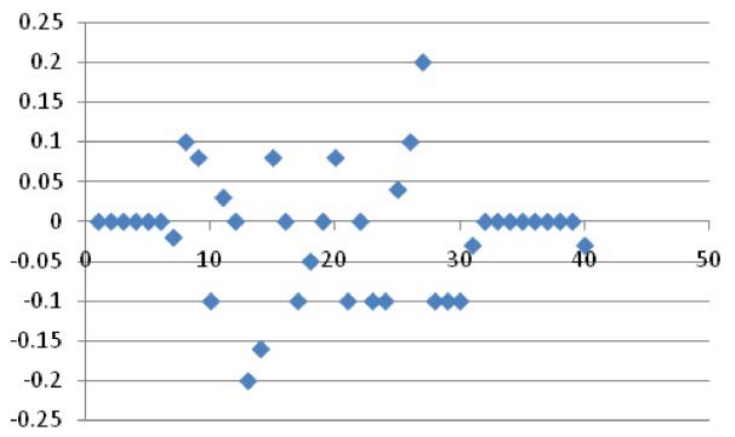

3D-PHT

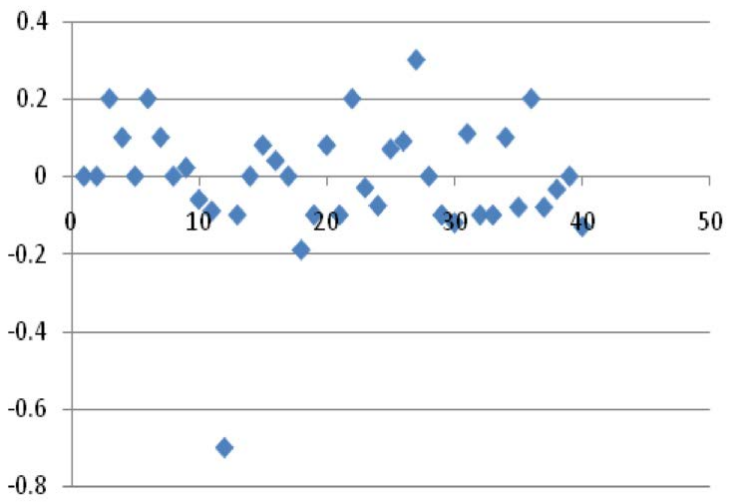

Figure 4: $4 a, 4 b$ and $4 c$ Showing scatter plot diagram Showing difference between various methods of MVA calculation and PHT method by difference in difference method.
Table 3 : Showing difference between various methods of MVA calculation and PHT method by difference in difference method.

\begin{tabular}{|llll} 
& 2D-PHT & 3D Xplane-PHT & 3D- PHT \\
\hline SD & 0.142307 & 0.075717 & 0.157504 \\
\hline mean(-2sd) & -0.29961 & -0.16593 & -0.32246 \\
\hline mean(+2sd) & 0.269614 & 0.136934 & 0.307558 \\
\hline Difference & -0.56923 & -0.30287 & -0.63002 \\
\hline
\end{tabular}

\section{DISCUSSION}

To define the best therapeutic strategy in patients with RMVS, clinical data and accurate measurements of MVA are necessary. Doppler-based methods are heavily influenced by hemodynamic variables, left ventricular hypertrophy, and associated valvular disease. ${ }^{10-13}$ Accordingly, methods based on direct measurement of valvular orifice should be more accurate. To date, direct measurements of the MVA area can only be performed using planimetry traced on 2DE images. However, this method has multiple limitations, the major one being the correct image plane orientation. Studies have shown good correlation between Doppler PHT and invasive method of MVA calculation by Gorlin formula, therefore we took Doppler PHT as the gold standard method of MVA for our study. ${ }^{14}$ Live $3 \mathrm{DE}$ improves the operator's ability to perform a well-oriented and accurate MVA planimetry. ${ }^{6,7}$ With the recent advent of Live $3 \mathrm{DE}$, many of these limitations have been overcome. Live $3 \mathrm{DE}$ allows evaluation of the MVA "en-face." Additionally, flexibility, rotation, and orientation of the mitral valve to the desired plane are easy and independent of the orientation of the acoustic window where image acquisition is done. There are several studies in the past showing good agreement between $3 \mathrm{DE}$ and invasive method by Gorlin formula. ${ }^{15,16}$ But there are also few studies which doesn't show good correlation between 3DE and Doppler 2D methods. ${ }^{17}$ Our study showed that all methods for calculation of MVA are valid but there was not a good correlation $2 \mathrm{DE}$ and $3 \mathrm{DE}$ with Doppler PHT method and intraobserver and interobserver variability was more for $2 \mathrm{DE}$ and $3 \mathrm{DE}$. There are few studies to support our results that show that MVA can be slightly underestimated with $3 \mathrm{DE} .{ }^{18}$

Our study validates that $3 \mathrm{D}$ Xplane is the most accurate echocardiography parameter for measuring MVA using non invasively determined PHT data as the gold standard. Xplane technique has an edge over conventional 2D TTE in that it displays mitral leaflet separation and corresponding planimetered MVA in same bisected image. The study done by RK Gokhroo et al also showed that MVA by 3D Xplane method is simple and reliable method of MS severity. ${ }^{18}$ Independent analysis of the results from both observers showed that $3 \mathrm{D}$ Xplane planimetry is the most reproducible, feasible and accurate method to measure MVA. Furthermore, 3D Xplane measurements have less interand intraobserver variability and provide the best interobserver agreement for MVA measurement.

\section{Study limitations}

One limitation is that the echocardiography delineation of the MVA is always dependent on the quality of the image. MVA measurements obtained from 3DE were not compared with measurements derived from cardiac catheterization and 3D TEE which could have more better validated our results.

\section{Clinical implications}

3D Xplane method can improve the assessment of Rheumatic MVA and severity in patients with discordant results between 
different methods and in clinical scenarios where these methods are known as not useful (i.e., the early postvalvuloplasty period). Thus, it is able to replace other non-invasive methods and make invasive evaluation unnecessary.

\section{CONCLUSIONS}

3D Xplane echocardiography is feasible, accurate, and highly reproducible for estimating MVA in patients with RMVS. Compared with other currently used modalities,3D Xplane echocardiography has the best agreement with the other non invasive methods of MVA measurement. It was a single center observational study which needs to be extended to other high volume centers to further validate this observation.

\section{ABBREVIATIONS}

2DE Two dimensional Echocardiography

3DE Three dimensional Echocardiography

3D Xplane Three dimensional X plane

MVA Mitral valve area

MS Mitral Stenosis

PHT Pressure half-time

PLAX Parasternal long Axis

PTMC Percutaneous transmitral commissurotomy

RMVS Rheumatic mitral valve stenosis

TTE Transthoracic Echocardiography

\section{REFRENCES}

1. Nishimura RA, Rihal CS, Tajik AJ, et al: Accurate measurement of the transmitral gradient in patients with mitral stenosis: A simultaneous catheterization and Doppler echocardiographic study. J Am CollCardiol 1994;24: 152158.https://doi.org/10.1016/0735-1097(94)90556-8

2. Rifkin RD, Harper K, Tighe D: Comparison of proximal isovelocity surface area method with pressure halftimeandplanimetry in evaluation of mitral stenosis. J Am CollCardiol 1995;26:458-465.https://doi.org/10.1016/07351097(95)80023-A

3. Lee TY, Hsu TL, Tseng CJ, et al: Clinical applicability for the assessment of the valvular mitral stenosis severity with Doppler echocardiography and the proximal isovelocity surface area (PISA) method. Echocardiography 2004;21:1-6. https://doi.org/10.1111/j.0742-2822.2004.03057.x

4. Kawahara T, Yamagishi M, Seo H, et al: Application of Doppler color flow imaging to determine valve area in mitral stenosis. J Am CollCardiol 1991;18:85-92.https://doi. org/10.1016/S0735-1097(10)80223-2

5. Faletra F, Pezzano A Jr, Fusco R, et al. Measurement of mitral valvearea in mitralstenosis: four echocardiographic methods compared withdirect measurement of anatomic orifices. J Am CollCardiol 1996;28:1190-7.https://doi.org/10.1016/ S0735-1097(96)00326-9

6. Chen Q, Nosir YF, Vletter WB et al. Accurate assessment of mitral valve area in patients with mitral stenosis by threedimensional echocardiography. J Am SocEchocardiogr 1997;10:133-40.https://doi.org/10.1016/S08947317(97)70085-3

7. Baumgartner H, Hung J, Bermejo J, et al. Echocardiographic assessment of valve stenosis: EAE/ASE recommendations for clinical practice. J Am SocEchocardiogr. 2009;22:1-23. https://doi.org/10.1016/j.echo.2008.11.029

8. Smith MD, Wisenbaugh T, Grayburn PA et al. Value and limitations of Doppler pressure half-time in quantifying mitral stenosis: a comparison with micromanometer catheter recordings. Am Heart J 1991;121:480-8.https://doi. org/10.1016/0002-8703(91)90715-T

9. Lang RM, Badano LP, Tsang $W$, et al. EAE/ASE recommendations for image acquisition and display using three-dimensional echocardiography. J Am SocEchocardiogr. 2012;25:3-46.https://doi.org/10.1016/j.echo.2011.11.010

10. Nakatani S, Masuyama T, Kodama K et al. Value and limitations of Doppler echocardiography in the quantification of stenotic mitral valve area: comparison of the pressure half-time and the continuity equation methods. Circulation 1988;77:78-85.https://doi.org/10.1161/01.CIR.77.1.78

11. Karp K, Teien D, Bjerle P et al. Reassessment of valve area determinations in mitral stenosis by the pressure half-time method: impact of left ventricular stiffness and peak diastolic pressure difference. J Am CollCardiol1989;1:594-9.https:// doi.org/10.1016/0735-1097(89)90599-8

12. Flachskampf FA, Weyman AE, Gillam L et al. Aortic regurgitation shortens Doppler pressure half-timein mitral stenosis: clinical evidence, in vitro simulation and theoretic analysis. J Am CollCardiol1990;16:396-404.https://doi. org/10.1016/0735-1097(90)90592-D

13. Fredman CS, Pearson AC, Labovitz AJ, et al: Comparison of hemodynamic pressure half-time method and Gorlinformula with Doppler and echocardiographic determinations of mitral valve area in patients with combined mitralstenosis and regurgitation. Am Heart J 1990;119:121-129https://doi. org/10.1016/S0002-8703(05)80091-2

14. Sugeng L, Weinert L, Lammertin G et al . Accuracy of mitral valve area measurements using transthoracic rapid freehand 3-dimensional scanning: comparison with noninvasive and invasive methods. J Am SocEchocardiogr. 2003;16:12921300.https://doi.org/10.1067/j.echo.2003.07.005

15. Zamorano J, Cordeiro P, Sugeng L et al. Real-time threedimensional echocardiography for rheumatic mitral valve stenosis evaluation: an accurate and novel approach. J Am CollCardiol. 2004;43:2091-2096.https://doi.org/10.1016/j. jacc.2004.01.046

16. Woon-Seok Kang, Jae Won Choi, Joo-Eun Kang et al. Determination of mitral valve area with echocardiography, using intra-operative 3 -dimensional versus intra- \& postoperative pressure half-time technique in mitral valve repair surgeryJ Cardiothorac Surg. 2013; 8: 98.https://doi. org/10.1186/1749-8090-8-98

17. Binder TM, Rosenhek R, Porenta G et al. Baumgartner H. Improved assessment of mitral valve stenosis by volumetric real-time three-dimensional echocardiography. J Am CollCardiol. 2000 Oct; 36( 4):1355-61.https://doi. org/10.1016/S0735-1097(00)00852-4

18. Gokhroo R K, Ranwa B L, Kishore K, Preeti K. 3D Xplane Echocardiographic Technique for Validation of Mitral Leaflet Separation to Assess Severity of Mitral Stenosis. Echocardiography. 2016 Jun; 33(6): 896-901https://doi. org/10.1111/echo.13183

19. Xie MX, Wang XF, Cheng TO et al. Comparison of accuracy of mitral valve area in mitral stenosis by real-time, threedimensional echocardiography versus two-dimensional echocardiography versus Doppler pressure half-time. Am J Cardiol. 2005;95:1496-1499. https://doi.org/10.1016/j. amjcard.2005.02.023

20. Thomas M. Binder, R Rosenhek, G Porenta et al. Improved Assessment of Mitral ValveStenosis by Volumetric RealTime Three-Dimensional Echocardiography. JACC Vol. 36, No. 4, 2000 October:1355-61https://doi.org/10.1016/ S0735-1097(00)00852-4

Cite this article as: Amit kumar. Agarwal, Deewakar Sharma, Sajan Gopal Baidya, et.al. Variability among 2D and 3D methods of calculating mitral valve area: a comparative study with pressure half time method. Nepalese Heart Journal 2017;12(2):13-17. http://dx.doi.org/10.3126/njh.v14i2.18497 